US Army Corps

of Engineers ${ }_{\circledast}$

Engineer Research and

Development Center

Environmental Consequences of Nanotechnologies

\title{
Determination of Contact Angle and Surface Tension of Nanomaterial Solutions by Optical Contact Angle System
}

Qihua Wu, Kathryn Kremer, Stephen Gibbons,

June 2019 and Alan J. Kennedy

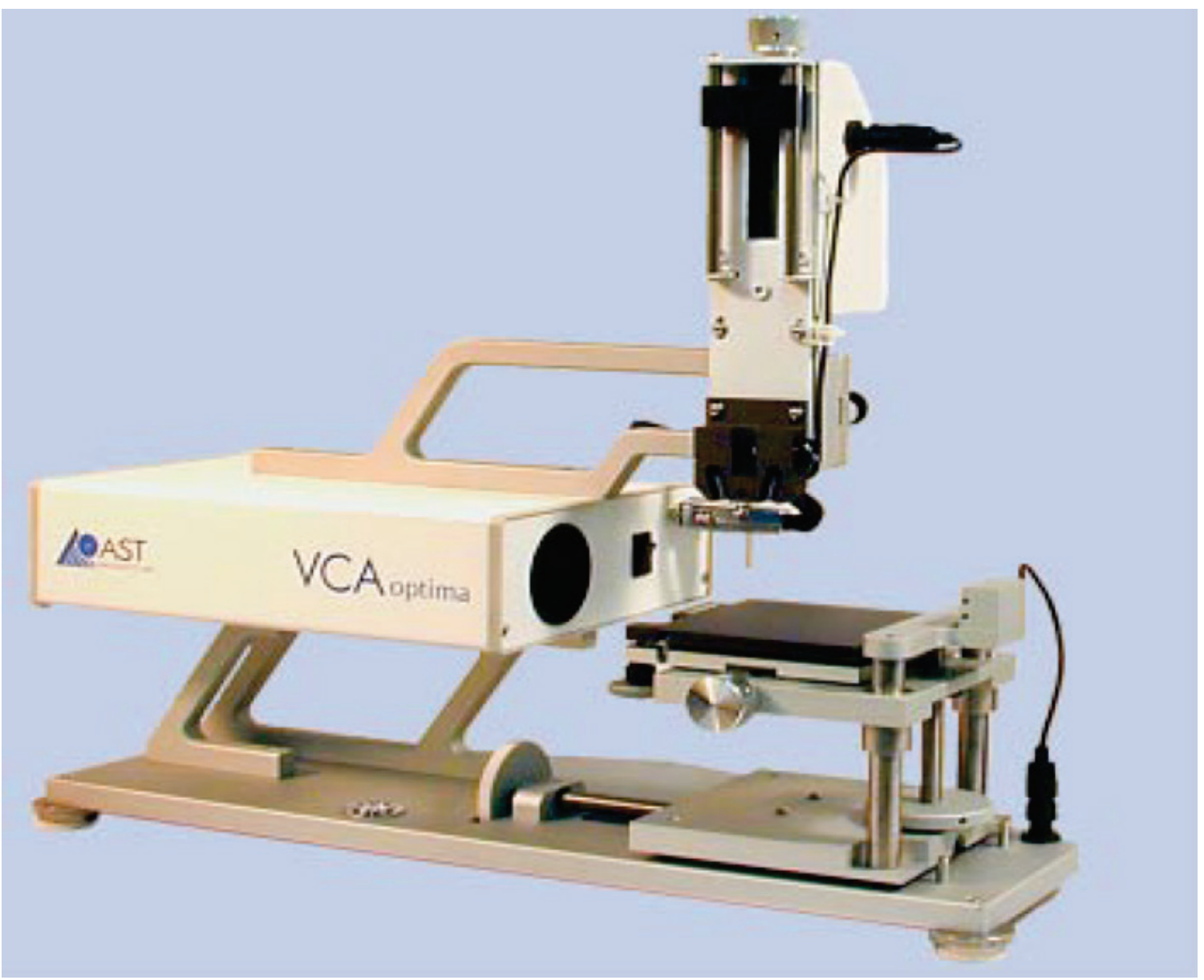


The U.S. Army Engineer Research and Development Center (ERDC) solves the nation's toughest engineering and environmental challenges. ERDC develops innovative solutions in civil and military engineering, geospatial sciences, water resources, and environmental sciences for the Army, the Department of Defense, civilian agencies, and our nation's public good. Find out more at www.erdc.usace.army.mil.

To search for other technical reports published by ERDC, visit the ERDC online library at http://acwc.sdp.sirsi.net/client/default. 


\section{Determination of Contact Angle and Surface Tension of Nanomaterials Solutions by Optical Contact Angle System}

Qihua Wu, Kathryn Kremer, and Stephen Gibbons

Brewer Science, Inc.

2401 Brewer Drive

Rolla, MO 65401

Alan J. Kennedy

U.S. Army Engineer Research and Development Center

Environmental Laboratory

3909 Halls Ferry Road

Vicksburg, MS 39180-6199

Final Report

Approved for public release; distribution is unlimited.

Prepared for Headquarters, U.S. Army Corps of Engineers

Washington, DC 20314-1000

Under Contract number W912HZ-15-2-0032, "Advancing Carbon NanomaterialsBased Device Manufacturing through Life Cycle Analysis, Risk Assessment and Mitigation." 


\section{Abstract}

Contact angle and surface tension measurements reveal wetting characteristics of materials, which are very essential for both fundamental research and manufacturing processes. Among many types of techniques, optical contact angle measurement is considered to be the most convenient method and has been widely used. This scientific operating procedure (SOP) provides general guidance for using an optical contact angle measurement system (e.g., an AST VCA Optima system), for determining contact angle and surface tension of nanomaterials in solution. Procedures and recommendations for sample preparation, instrument preparation, sample analysis, and results analysis are included. This procedure was tested on a variety of nanomaterial dispersions.

DISCLAIMER: The contents of this report are not to be used for advertising, publication, or promotional purposes. Citation of trade names does not constitute an official endorsement or approval of the use of such commercial products. All product names and trademarks cited are the property of their respective owners. The findings of this report are not to be construed as an official Department of the Army position unless so designated by other authorized documents. 


\section{Contents}

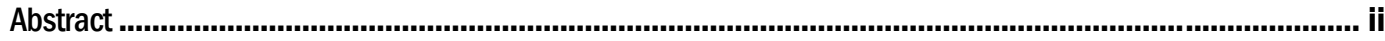

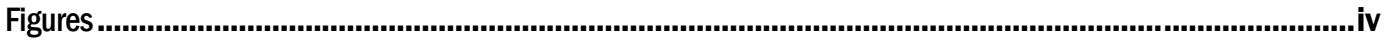

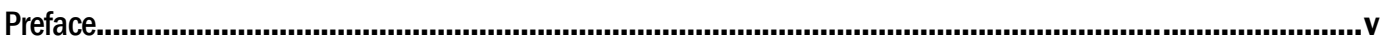

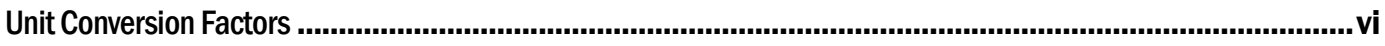

Acronyms and Abbreviations............................................................................................................vii

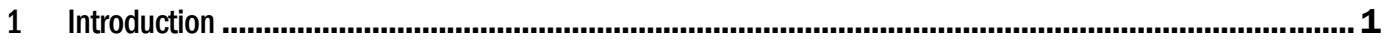

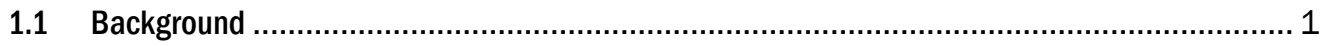

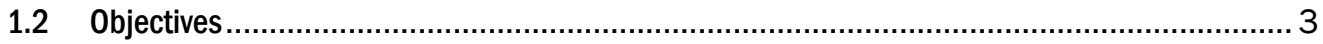

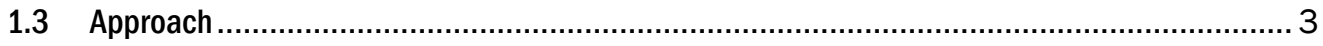

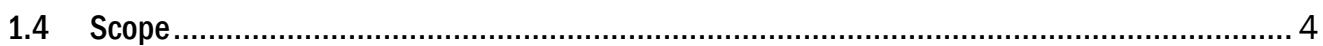

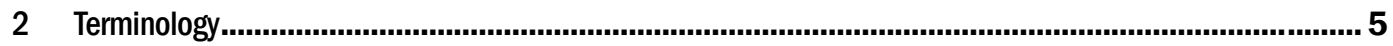

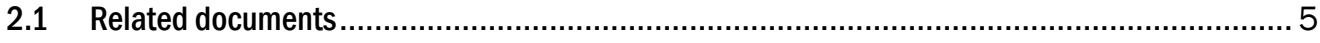

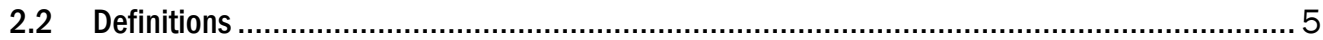

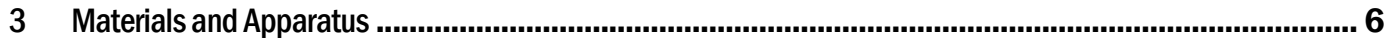

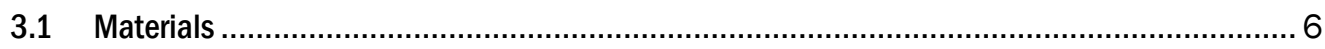

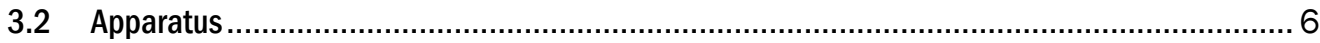

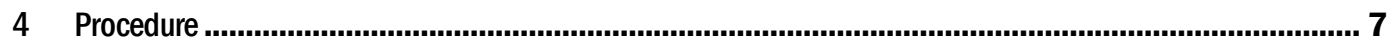

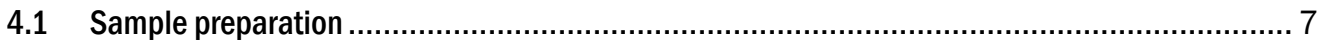

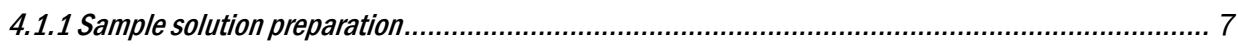

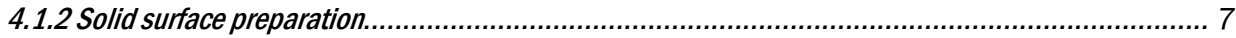

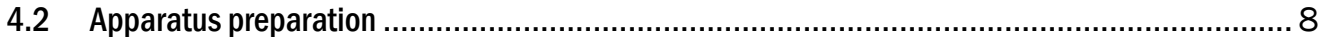

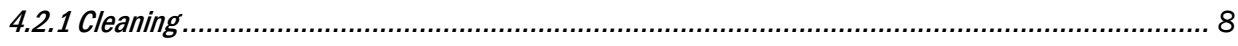

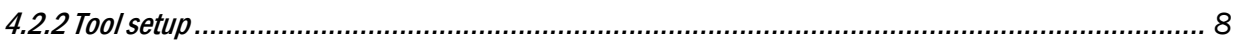

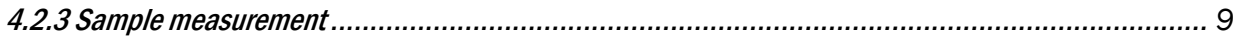

$5 \quad$ Reporting..............................................................................................................................12

5.1 Analysis of contact angle results....................................................................... 12

5.2 Calculation of solid surface tension .................................................................. 13

5.3 Determination of critical concentration for nanomaterial suspensions ............................... 13

5.4 Quality assurance (QA)/Quality control (QC) considerations ....................................... 14

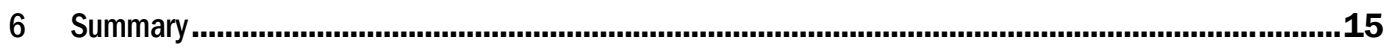

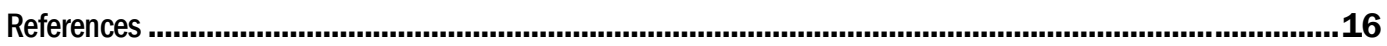

\section{Report Documentation Page}




\section{Figures}

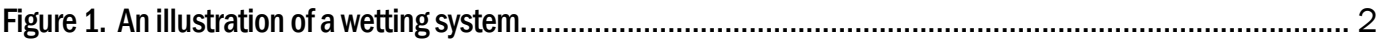

Figure 2. Analysis of liquid surface tension from pendant drop measurement. ............................................... 11

Figure 3. Example of liquid sample contact angle measurement on a silicon substrate.................................. 12

Figure 4. Example of surface tension versus concentration for determination of critical concentration. ..................................................................................................... 13 


\section{Preface}

This sscientific operating procedure (SOP) was developed under Task 1: "Materials evaluation and characterization" of the project "Advancing Carbon Nanomaterials-Based Device Manufacturing through Life Cycle Analysis, Risk Assessment and Mitigation.” This program was funded by the Engineer Research and Development Center (ERDC) of the U.S. Army Corps of Engineers (USACE), contract number W912HZ-15·2-0032. The program manager was Mr. Alan J. Kennedy. This work was directed by Mr. Rishi J. Patel, Senior Research Scientist at Missouri State University's Jordan Valley Innovation Center. This task was under the direct supervision of Dr. Wu-Sheng Shih of Brewer Science, Inc. The technical monitor was Dr. Elizabeth A. Ferguson.

The work was performed by the Environmental Risk Assessment Branch (EPR) of the Environmental Processes and Engineering Division (EPE), U.S. Army Engineer Research and Development Center - Environmental Laboratory (ERDC-EL). At the time of publication, Dr. William M. Nelson was Chief, CEERD-EPR, and Dr. Elizabeth A. Ferguson was the Technical Director. The Deputy Director of ERDC-EL was Dr. Jack E. Davis, and the Director was Dr.Ilker R. Adiguzel.

COL Ivan P. Beckman was Commander of ERDC, and Dr. David W. Pittman was the Director. 


\section{Unit Conversion Factors}

\begin{tabular}{|l|l|l|}
\hline Multiply & By & To Obtain \\
\hline angstroms & 0.1 & nanometers \\
\hline degrees (angle) & 0.01745329 & radians \\
\hline degrees Fahrenheit & $(\mathrm{F}-32) / 1.8$ & degrees Celsius \\
\hline microinches & 0.0254 & micrometers \\
\hline
\end{tabular}




\section{Acronyms and Abbreviations}

\begin{tabular}{|l|l|}
\hline Acronym & \multicolumn{1}{|c|}{ Meaning } \\
\hline CCD & Charge Coupled Device \\
\hline DI & Deionized \\
\hline DoD & Department of Defense \\
\hline EL & Environmental Laboratory \\
\hline EPE & Environmental Processes and Engineering \\
\hline EPR & Environmental Risk Assessment \\
\hline ERDC & Engineer Research and Development Center \\
\hline HPLC & High-Performance Liquid Chromatography \\
\hline IPA & Isopropyl alcohol \\
\hline MI & Methylene iodide \\
\hline SOP & Scientific Operating Procedure \\
\hline QA & Quality Assurance \\
\hline QC & Quality Control \\
\hline USACE & U.S. Army Corps of Engineers \\
\hline
\end{tabular}




\section{Introduction}

This document provides a scientific operating procedure (SOP) for determining the contact angle and surface tension of nanomaterials in solution, or nanomaterial-coated substrates, using an optical contact angle measurement system.

Wetting information is essential to many applications and processes such as petroleum oil recovery, coating and printing, lubrication, development of biocompatible surfaces, membranes, and many others (Prabhu et al. 2009; Zhao et al. 2010; Wang et al. 2011; Sakai et al. 2008; Son et al. 2008; Perelaer et al. 2009). In a wetting system, the contact angle is commonly used to quantify the degree of wettability of a solid surface by a liquid phase. Measured contact angle values can be used for the assessment of another important parameter, surface tension. Surface tension represents the intermolecular forces that contact the surface, providing another quantification of the wetting characteristics of a material. Knowing a material's surface tension is critical for designing and controlling a wetting process. For nanomaterial research and applications, characterizations of contact angle and surface tension have also played important roles (Rauscher and Dietrich 2008; Liu and Webster 2007). The following section will discuss fundamentals of contact angle and surface tension as they relate to the wetting phenomenon, including the introduction of techniques for contact angle and surface tension measurements. Specifically, this discussion includes how the contact angle of a liquid sample drop on a standard substrate can be used for the wettability study, while the contact angle of a standard solution drop on a sample surface provides the solid's surface tension. The surface tension of a liquid sample can be obtained by a pendant drop measurement.

\subsection{Background}

The contact angle of a liquid wetting a solid is the angle formed by a tangent to the liquid and the surface of the solid measured at the boundary where the three phases, liquid, solid, and fluid (gas) meet. Figure 1 shows an example of a wetting system consisting of a liquid drop on a flat solid surface, all immersed in a fluid (gas). 
Figure 1. An illustration of a wetting system.

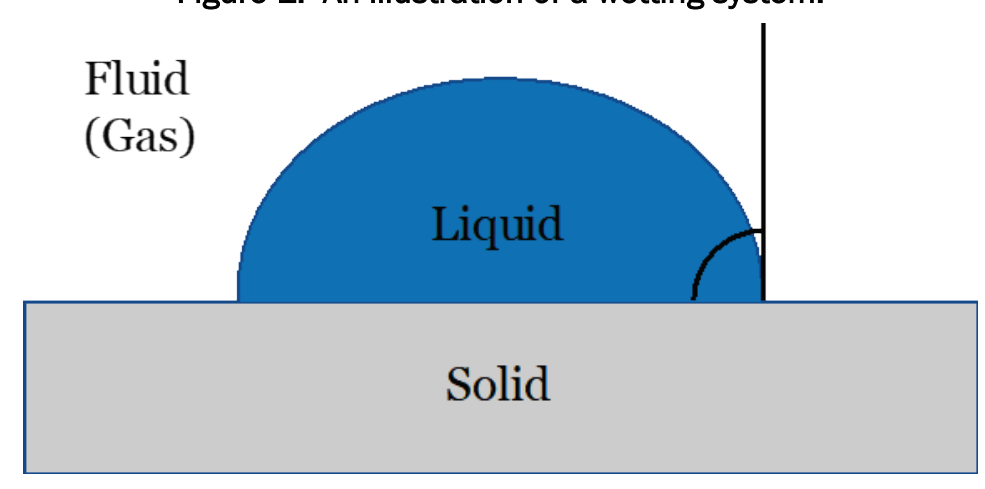

The measured contact angle usually indicates the degree of wetting. A contact angle of less than $90^{\circ}$ indicates a greater wetting of the surface (higher wettability), and the liquid phase will tend to spread on the solid surface. In contrast, a large contact angle (greater than $90^{\circ}$ ) corresponds to a lower wettability, and the liquid phase will minimize contact with the solid surface.

The contact angle of a liquid drop on a solid surface was first defined by Young's equation (Young 1805)

$$
\cos \theta_{Y}=\frac{\sigma_{s f}-\sigma_{s l}}{\sigma_{l f}}
$$

Where, $\cos \theta_{Y}$ is the Young's contact angle (YCA), and $\sigma_{s f}, \sigma_{s l}$, and $\sigma_{l f}$ represent the solid-fluid, solid-liquid, and liquid-fluid interfacial tensions, respectively. Young's equation was developed assuming a perfectly flat and smooth surface, which is referred to as an ideal surface. The obtained YCA depends only on the physical-chemical properties of the three phases and represents the state of the solid-liquid-fluid system with the minimal Gibbs energy (Marmur 2006).

Many techniques have been developed for measuring the contact angle of a material (Bigelow et al. 1946; Taggart et al. 1929; Adam and Jessop 1925; Wilhelmy 1863; Good and Pascheck 1978; Neumann and Good 1979). The most widely used technique is a direct optical method using a telescopegoniometer, which was first developed by Bigelow et al. (1946). The apparatus usually consists of a pipette or syringe for dispensing a sample drop onto a horizontal stage that holds the solid surface. A camera is focused on, and aligned with, the contact point between the sample drop and the solid surface. The syringe is controlled by a motor to form the designated drop size while the image is recorded by the camera. This 
optical method has many advantages. It is simple and fast, requires a small sample size (for both liquid and solid), and is capable of studying advancing, receding, or dynamic contact angles (Kwok et al. 1996). There are also some limitations of this technique, for instance, the measurement reproducibility greatly depends on the operation consistency. Most telescope-goniometer instruments still rely on the operator to determine the tangent line of the droplet; thus, there could be significant variation among different operators. Another limitation is that ultrasmall contact angles (usually less than $15^{\circ}$ ) could be difficult to measure depending on the resolution of the camera and sample drop size (Brandon et al. 2003; Letellier et al. 2007). Other measurement methods are used based on measurements of contact angles with different sample geometric forms: the captive bubble method (Taggart et al. 1929), the tilting plate method (Adam and Jessop 1925), the Wilhelmy balance method (Wilhelmy 1863), and the capillary tube method (Good and Pascheck 1978). Among these methods, the goniometer method is still considered to be the most convenient and applicable (Neumann and Good 1979).

Once the contact angle is measured, another important parameter, surface tension, can be estimated. Surface tension results from the intermolecular forces that contact a material's surface and define wetting characteristics. In most applications, the liquid used for measuring the contact angle may not represent all liquid samples in practice. If the solid surface tension is known, the wetting process and contact angle of the liquid with known surface tension can be estimated. The solid surface tension is commonly measured by testing the contact angle of "standard" solutions with known surface tension on the solid surface (Kwok and Neumann 2003). The solid surface tension can then be calculated by one of many methods (e.g., the Owens-Wendt equation (Kwok and Neumann 2003)).

\subsection{Objectives}

The objective of this SOP is to define the general procedure for measuring contact angles to determine wettability and surface tension of carbonbased nanomaterial solutions by using optical contact angle metrology.

\subsection{Approach}

This SOP is focused on the test of nanomaterial suspensions and solutions. Although some parts of the procedure have things in common with conventional methods, several sections specifically apply to nanomaterials. 
Preparing samples of nanomaterials solutions requires extra care, as the interactions between nanomaterial particles could change the interface of liquid/solid and liquid/gas. The data analysis for nanoamterials may involve extra steps to determine the critical concentration of nanomaterials.

\subsection{Scope}

This SOP should be used for determining the contact angle and surface tension of nanomaterial solutions by using an optical contact angle measurement system. The following types of measurements are included: (1) contact angle measurement of a nanomaterial solution drop on a target substrate for the wettability study, (2) contact angle measurement of a standard solution drop on a nanomaterial-coated surface to measure solid surface tension, and (3) pendant drop measurement of a nanomaterial solution to measure liquid surface tension.

This SOP does not provide a detailed method for specific kinds of nanomaterials. It provides general guidance for most nanomaterial suspensions/solutions as well as recommended sample preparation/data analysis steps specific to nanomaterials. The procedure for operating an AST VCA Optima instrument is demonstrated in this example. 


\section{Terminology}

\subsection{Related documents}

- ASTM D7334-08 (2013) Standard Practice for Surface Wettability of Coatings, Substrates and Pigments by Advancing Contact Angle Measurement.

- ASTM D7490-13 (2013) Standard Test Method for Measurement of the Surface Tension of Solid Coatings, Substrates and Pigments by Contact Angle Measurements.

- ASTM D1331-14 (2014) Standard Test Method for Measurement of the Surface Tension of Solid Coatings, Substrates and Pigments by Contact Angle Measurements.

- ISO 19403 (2017) Paints and Varnishes - Wettability

- Instrument operation manual provided by instrument manufacturers (i.e., AST VCA Optima).

\subsection{Definitions}

- Contact angle: the interior angle between the tangent to the liquidsolid interface and the tangent to the liquid-gas interface when a liquid drop rests on a solid surface.

- Surface tension (also refers to surface energy): the molecular cohesive force that contracts a surface.

- Solid surface tension: the surface tension of a solid surface. Generally, this parameter is not measured directly, but it can be calculated based on contact angles of liquids with known surface tension (as described in this SOP and ASTM D7940-13).

- Nanoparticle: an ultrafine object (particle) with its dimensions between 1 and $100 \mathrm{~nm}$.

- Nanoparticle suspension: a heterogeneous liquid mixture in which nanoparticles are suspended in solution but not dissolved. 


\section{Materials and Apparatus}

\subsection{Materials}

- Nanomaterial solution

- Substrate or solid surface (e.g., glass slide, silicon substrate)

- Nanomaterial-coated sample substrate

- Deionized (DI) water

- Compressed dry air or nitrogen

- Isopropyl alcohol (IPA)

- Methylene iodide (MI)

- Syringes (glass syringes should be used for samples containing organic solvents)

- Syringe needles

\subsection{Apparatus}

An instrument consisting of an adjustable light source, a sample stage to hold the test specimen, and a digital camera for viewing and recording of the drop on the specimen is required. 


\section{Procedure}

The procedure described below covers the generic practice for measuring contact angle and surface tension of nanomaterials in an aqueous suspension. Some sections use the operation of an AST VCA Optima instrument in the demonstration.

\subsection{Sample preparation}

The measurement should require minimal or no sample preparation. The main purpose of any sample/specimen preparation is to ensure the homogeneity of the sample and to protect the apparatus.

\subsubsection{Sample solution preparation}

Use a highly pure solvent (e.g., DI water of a grade suitable for highperformance liquid chromatography (HPLC)) for sample preparation, as sample purity will greatly affect test results. The sample solution should be well mixed by an appropriate method to ensure homogeneity. There are many different types of techniques for dispersing nanomaterials into solution (Taurozzi et al. 2012), but the details are not discussed in this SOP. The sample can be filtered to remove dust particles or bubbles if necessary. Surfactant or any other reagent that could change the surface tension of the sample should be avoided.

When the concentration of nanomaterials is high enough (above a certain critical concentration), the contact angle and surface tension of sample droplet measured using an optical method may become largely dependent on particle concentrations. This phenomenon was explained as being caused by the van der Waals force of the particles at the interfaces liquid/gas and liquid/solid (Tanvir and Qiao 2012; Vafaei et al. 2006). For that reason, it is recommended to test the sample at relatively low concentration (generally less than $0.1 \%$ wt). For the sample with a relatively high particle concentration, prepare a series of samples with different concentrations, and test the contact angle/surface tension of each sample.

\subsubsection{Solid surface preparation}

The solid surface to be tested should be rigid, flat, and smooth to obtain the best test results. If the material is flexible, affix it to the stage with 
double-sided tape or clips. Use an appropriate procedure to clean the solid surface to avoid any dust or contamination. Avoid using a bare hand to touch the surface of the specimen, as the hand's natural oils will greatly affect test results. Also, avoid using other surface-active substances/reagents. If the test specimen is too large to fit on the stage, the test specimen should be cut to a size appropriate for the instrument.

\subsection{Apparatus preparation}

This section describes the general procedure for apparatus preparation using an AST VCA Optima instrument as the example.

\subsubsection{Cleaning}

All components, especially the sample dispensing system, the syringe, and the needle, should be cleaned and free of contaminants. The use of acetone or IPA rinsing followed by DI water rinsing is recommended. If necessary, use dry air or nitrogen to dry the components. Place the components back into position on the apparatus.

\subsubsection{Tool setup}

Switch on the instrument power and check the calibration/certification. Ensure the camera is focused and calibrated. Ensure the instrument is operated at the ambient temperature of $22^{\circ} \pm 3{ }^{\circ} \mathrm{C}$ and a stable relative humidity (preferably $40 \%$ or higher during measurement), unless other conditions have been established to meet a specific need. Ensure the stage is leveled and make necessary adjustments according the manufacturer's instructions.

\subsubsection{Syringe setup}

Ensure the syringe is cleaned before filling with test samples. Use an appropriate syringe and needle for the sample and standards. For sample or standard solutions with high viscosity, such as glycerol, use a largerdiameter syringe needle. Never cross-use the syringe and needle to avoid cross-contamination. It is recommended to flush the syringe and needle with sample/standard solutions before loading the syringe. Place a small container or blank substrate appropriately to catch droplets while loading the syringe, then load the syringe and needle onto the apparatus. Secure the syringe and adjust the position of the stage so that a droplet can be caught by the target solid surface. 


\subsubsection{Software setup}

Open the control software and set up the experimental parameters. Select the type of syringe/needle that is in use. Set the droplet size and dispense speed to appropriate values. Usually, the measurement time needs to be within 30 seconds to minimize sample evaporation.

Example experimental parameters for the VCA instrument are as follows:

- Sample delay: 1.0 second

- Dispense speed: medium

- Syringe size: $10 \mathrm{~mL}$

- Droplet size: 1.0 5.0 $\mu \mathrm{L}$.

\subsubsection{Sample measurement}

\subsubsection{Contact angle measurement}

This procedure is applicable for the following types of measurements:

1. Measuring the contact angle of an unknown liquid on a solid surface, for studying of wetting behavior, and

2. Measuring the contact angle of a known (standard) liquid on an unknown solid surface, for determining the solid surface tension (surface energy).

Place the solid surface specimen on the stage and ensure it is horizontal and flat. If necessary, use clips to secure the specimen in place. Open the light source and set the tip of the needle at the appropriate distance from the solid surface according to the instrument manufacturer's instructions. Start the syringe pump to deposit a droplet of test liquid on the solid surface. If necessary, adjust the focus of the camera and capture the image rapidly. Generally, water or aqueous contact angles must be measured within 30 seconds to avoid changes in the drop size due to evaporation. Then move the stage horizontally to a new area of the solid surface and make two additional measurements. The software should automatically calculate the contact angle on both edges. If the contact angles on two edges are significantly different, the test result should be discarded and another measurement should be made. For a series of samples with various concentrations, repeat above steps for each sample. 
To determine solid surface tension, perform contact angle measurements of at least two standard solutions on the sample specimen. DI water and MI are commonly used. Collect at least three sets of results for each standard liquid used.

\subsubsection{Pendant drop measurement}

Some optical contact angle meters are also capable of performing pendant drop measurements. A pendant drop measurement is a method where a liquid drop is suspended from a syringe needle, normally within a gaseous phase. The shape of the drop depends on the surface tension, interfacial tension, and gravity. Also, the surface tension of the liquid sample can be determined using the shadow image of the pendant and drop shape analysis provided by the instrument software. For a series of samples with various concentrations, repeat the above steps for each sample.

The main theory of the pendant drop measurement is based on the YoungLaplace equation (Equation 2)

$$
\Delta p=\sigma \cdot\left(\frac{1}{r_{1}}+\frac{1}{r_{2}}\right)
$$

Where, $\Delta p$ is the pressure difference between the inside and the outside surface of the droplet (curved surface), and $r_{1}$ and $r_{2}$ are the main radii of curvature of the droplet. The shape of the pendant drop and radii of curvature are affected by gravity (droplet weight) and hydrostatic pressure (surface tension). For most optical contact angle meters, only the droplet size needs to be controlled to capture the image of the pendant drop. Also, the software should be capable of processing the obtained images (Figure 2). 
Figure 2. Analysis of liquid surface tension from pendant drop measurement.

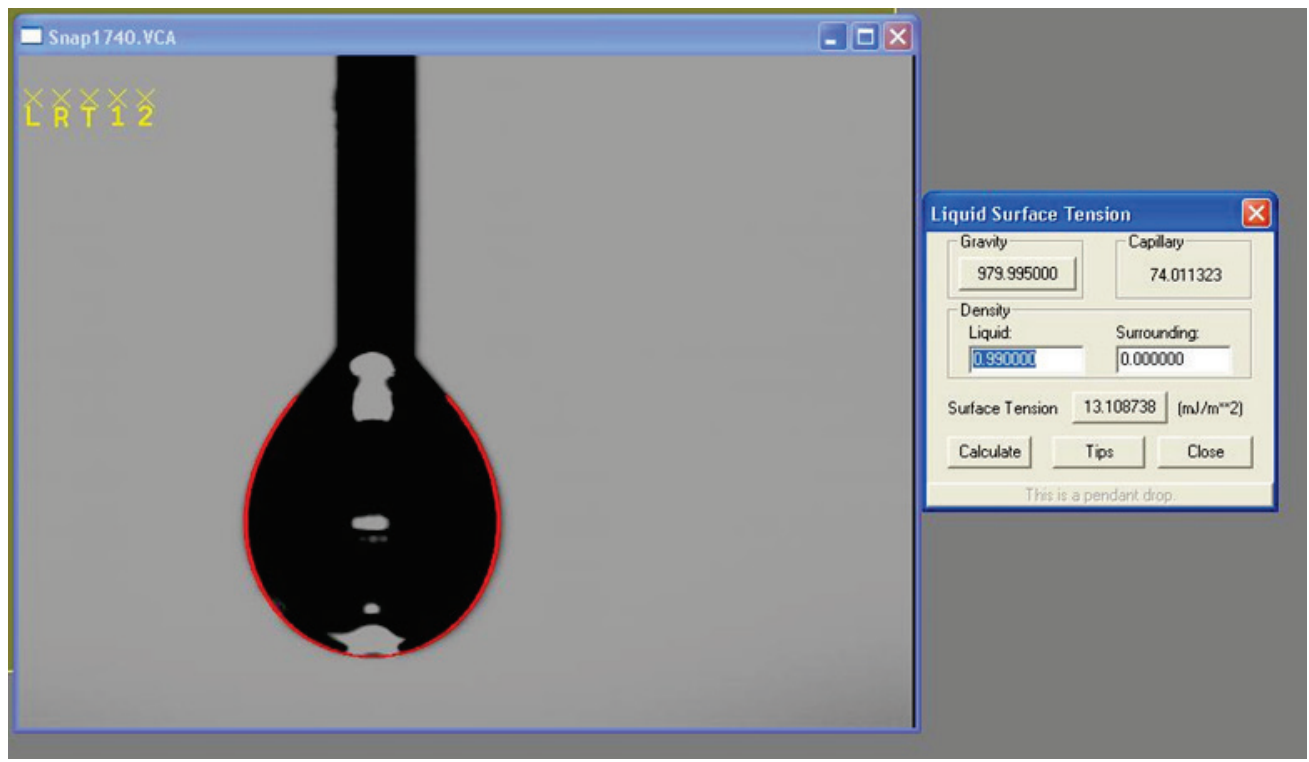




\section{Reporting}

\subsection{Analysis of contact angle results}

The contact angle of a liquid droplet on the substrate can be determined from the image captured by a charge coupled device (CCD) camera. Some software may have an auto-calculation function, but reviewing manual calculation is necessary in case auto-calculation is not accurate or is not available. To determine the tangent line and the substrate surface, the operator usually needs to define the shape of the droplet. Figure 3 provides an example of a liquid sample on a silicon substrate. To define the droplet, place the markers around the interface of the droplet. Note that each droplet will have two contact angle values, from the left side and the right side. The average of the left and right contact angles can be reported as the result. However, it is recommended to perform at least three individual measurements for each sample and report the average to minimize operation variations.

Figure 3. Example of liquid sample contact angle measurement on a silicon substrate.

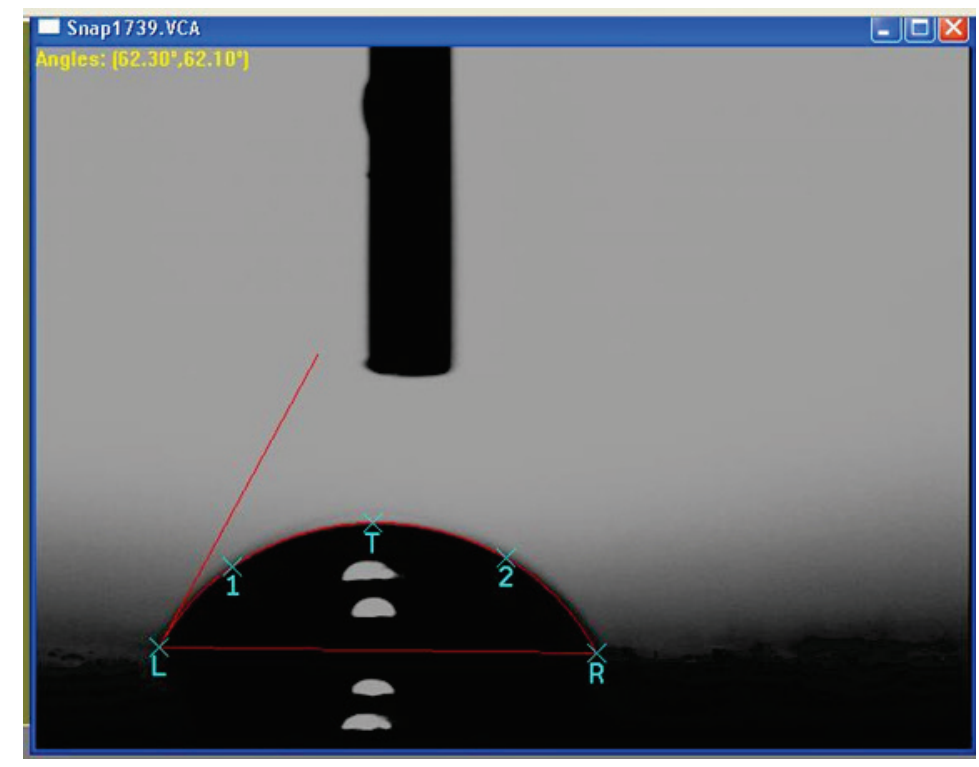

Obtained contact angle results can be used to determine the wettability of the liquid on target substrate. A low contact angle (usually less than $90^{\circ}$ ) is considered to be a favorable wetting, as the liquid will spread over the substrate. On the other hand, a contact angle greater than $90^{\circ}$ indicates the wetting is unfavorable. 


\subsection{Calculation of solid surface tension}

To determine the surface tension and surface energy of an unknown solid substrate, contact angles of standard solutions need to be measured. Most software for contact angle meters can calculate the solid surface tension from contact angle results of standard solutions. There are several different models developed for determining solid surface energy from liquid contact angles, such as the Zisman theory (Zisman 1964), the Owens-Wendt equation (Owens and Wendt 1969), and the Fowkes theory (Fowkes 1964). Refer to ASTM D7490-13 for a detailed procedure to calculate the solid surface tension based on the Owens-Wendt-Kaelble equation.

\subsection{Determination of critical concentration for nanomaterial suspensions}

As mentioned previously, surface tension and contact angle of some nanomaterials suspensions may become concentration-dependent when the concentration is high enough (referred as the critical concentration). To determine the critical concentration of a nanomaterial suspension, a series of samples with different concentrations are measured, and the surface tension at each concentration should be calculated (see section 4.2.3.2). Plot a smooth scattered line of surface tension verus the logarithm of concentration to the base 10. If the nanomaterial does have a critical concentration, a curve similar to Figure 4 will be obtained. Use the two tangent lines of the curve to determine the critical concentration.

Figure 4. Example of surface tension versus concentration for determination of critical concentration.

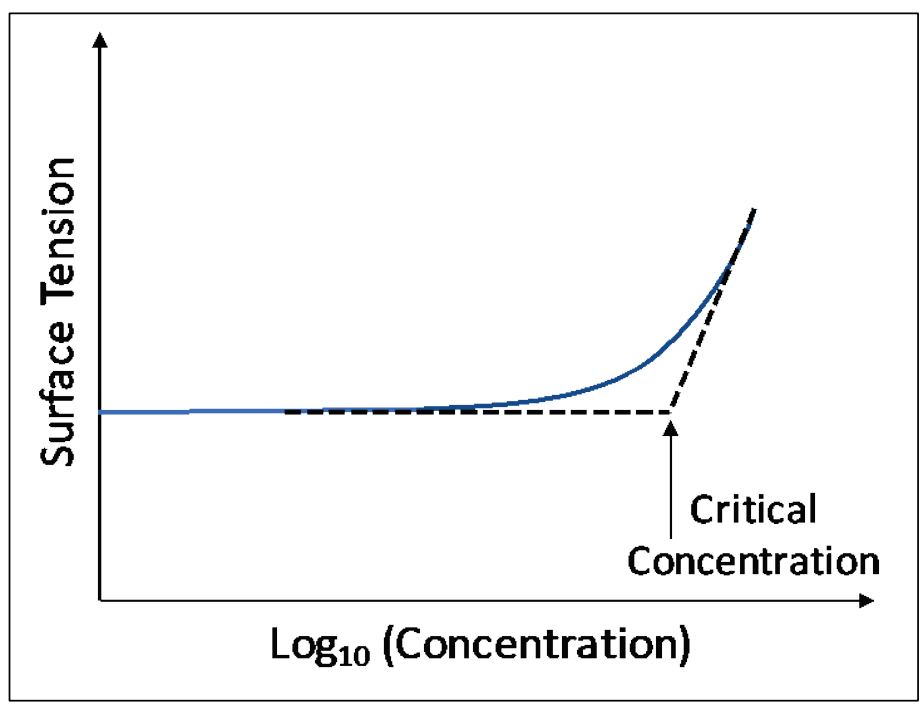




\subsection{Quality assurance (QA)/Quality control (QC) considerations}

Perform at least three replicated measurements for each sample. Standard deviation or percentage standard deviation for each sample from multiple measurements should be calculated to determine the repeatability. 


\section{Summary}

This SOP discusses a general procedure for using an optical contact angle measurement system, such as an AST VCA Optima system, to measure contact angles and surface tensions of nanomaterial-related samples. Sample preparation, instrument preparation, sample analysis, and results analysis are included in the procedure. The optical contact angle measurement technique has been widely used in many industrial and research areas, as contact angle and surface tension provide wetting characteristics of materials. 


\section{References}

Adam, N. K., and G. Jessop. 1925. CCL.-Angles of contact and polarity of solid surfaces. Journal of the Chemical Society, Transactions 127:1863-1868. doi: 10.1039/CT9252701863.

ASTM International. 2014. Standard Test Methods for Surface and Interfacial Tension of Solutions of Paints, Solvents, Solutions of Surface-Active Agents, and Related Materials. ASTM D1331-14. West Conshohocken, PA: ASTM International. doi: 10.1520/D1331-14.

ASTM International. 2013. Standard Practice for Surface Wettability of Coatings, Substrates and Pigments by Advancing Contact Angle Measurement. ASTM D7334-08. West Conshohocken, PA: ASTM International. doi: 10.1520/D7334.

ASTM International. 2013. Standard Test Method for Measurement of the Surface Tension of Solid Coatings, Substrates and Pigments using Contact Angle Measurements. ASTM D7490-13, West Conshohocken, PA: ASTM International. doi: 10.1520/D7490.

Bigelow, W. C., D. L. Pickett, and W. A. Zisman. 1946. Oleophobic monolayers: I. Films adsorbed from solution in non-polar liquids. Journal of Colloid Science 1(6):513538. https://doi.org/10.1016/0095-8522(46)90059-1.

Brandon, S., N. Haimovich, E. Yeger, and A. Marmur. 2003. Partial wetting of chemically patterned surfaces: The effect of drop size. Journal of Colloid and Interface Science 263(1):237-243. https://doi.org/10.1016/So021-9797(03)00285-6.

Fowkes, F. M. 1964. Attractive forces at interfaces. Industrial and Engineering Chemistry 56(12):40-52. doi: 10.1021/ie50660a008.

International Organization for Standardization (ISO). 2017. Paints and Varnishes Wettability - Part 4: Determination of the Polar and Dispersive Fractions of the Surface Tension of Liquids from an Interfacial Tension. ISO 19403-4. Geneva, Switzerland: International Organization for Standardization.

Kwok, D. Y., R. Lin, M. Mui, and A. W. Neumann. 1996. Low-rate dynamic and static contact angles and the determination of solid surface tensions. Colloids and Surfaces A: Physicochemical and Engineering Aspects 116(1-2):63-77. https://doi.org/10.1016/0927-7757(96)03590-X.

Kwok, D. Y., and A. W. Neumann 2003. Contact Angle, Wettability and Adhesion, Vol. 3. Boca Raton, FL: CRC Press.

Letellier, P., A. Mayaffre, and M. Turmine. 2007. Drop size effect on contact angle explained by nonextensive thermodynamics. Young's equation revisited. Journal of Colloid and Interface Science 314(2): 604-614. https://doi.org/10.1016/j.jcis.2007.05.085.

Liu, H., and T. J. Webster. 2007. Nanomedicine for implants: a review of studies and necessary experimental tools. Biomaterials 28(2): 354-369. https://doi.org/10.1016/j.biomaterials.2006.08.049. 
Marmur, A. 2006. Soft contact: Measurement and interpretation of contact angles. In Royal Society of Chemistry: Soft Matter 2(1):12-17. doi: 10.1039/B514811C.

Neumann, A. W. and R. J. Good. 1979. Surface and Colloid Science: Experimental Methods, Vol 11. New York, NY: Plenum Press.

Owens, D. K., and R. C. Wendt. 1969. Estimation of the surface free energy of polymers. Journal of Applied Polymer Science 13(8):1741-1747. https://doi.org/10.1002/app.1969.070130815.

Padday, John F., ed. Wetting, spreading, and adhesion: comprising papers (with discussions) presented to a symposium, organised by the Colloid and Surface Chemistry Group of the Society of Chemical Industry, held on 27-29 September 1976 at Loughborough University, Leicestershire. Academic Press, 1978.

Perelaer, J., C. E. Hendriks, A. W. M. de Laat, and U. S. Schubert. 2009. One-step inkjet printing of conductive silver tracks on polymer substrates. Nanotechnology 20(16): 165303. doi:10.1088/0957-4484/20/16/165303.

Prabhu, K. N., P. Fernades, and G. Kumar. 2009. Effect of substrate surface roughness on wetting behaviour of vegetable oils. Materials and Design 30(2):297-305. https://doi.org/10.1016/j.matdes.2008.04.067.

Rauscher, M., and S. Dietrich. 2008. Wetting phenomena in nanofluidics. Annual Review Materials Research 38:143-172. https://doi.org/10.1146/annurev.matsci.38.060407.132451.

Sakai, M., T Yanagisawa, A. Nakajima, Y. Kameshima, and K. Okada. 2008. Effect of surface structure on the sustainability of an air layer on superhydrophobic coatings in a water- ethanol mixture. Langmuir 25(1):13-16. doi: 10.1021/la802763h.

Son, Y., C. Kim, D. H. Yang, and D. J. Ahn. 2008. Spreading of an inkjet droplet on a solid surface with a controlled contact angle at low Weber and Reynolds numbers. Langmuir 24(6):2900-2907. doi: 10.1021/la702504v.

Taggart, A. F., T. C. Taylor, and C. R. Ince. 1929. Experiments with flotation reagents. American Institute of Mining and Metallurgical Engineers, Inc.

Tanvir, S. and Qiao, L., 2012. Surface tension of nanofluid-type fuels containing suspended nanomaterials. Nanoscale research letters, 7(1), p.226. https://doi.org/10.1186/1556-276X-7-226.

Taurozzi, J.S., Hackley, V.A., \& Wiesner, M.R. (2012). Preparation of nanoparticle dispersions from powdered material using ultrasonic disruption, Version 1.1. NIST special publication 1200(2). Gaithersburg, MD: National Institue of Standards and Technology (NIST), Material Measurement Laboratory. http://dx.doi.org/10.6028/NIST.SP.1200-2.

Vafaei, S., Borca-Tasciuc, T., Podowski, M.Z., Purkayastha, A., Ramanath, G. and Ajayan, P.M., 2006. Effect of nanoparticles on sessile droplet contact angle.

Nanotechnology, 17(10):2523. 
Wang, Y .Q., H. F. Yang, Q. G. Han, L. Fang, and S. R. Ge. 2011. Tribological and lubrication properties of sandblast-textured surfaces with varied roughness. In Advanced Materials Research 154:1019-1022. https://doi.org/10.4028/www.scientific.net/AMR.154-155.1019.

Wilhelmy, L. 1863. Ueber die Abhängigkeit der Capillaritäts-Constanten des Alkohols von Substanz und Gestalt des benetzten festen Körpers. Annalen der Physik 195(6):177-217. https://doi.org/10.1002/andp.18631950602.

Young, T. 1805. III. An essay on the cohesion of fluids. In Philosophical Transactions of the Royal Society of London 95:65-87. doi: 10.1098/rstl.1805.0005.

Zhao, X., M. J. Blunt, and J. Yao. 2010. Pore-scale modeling: Effects of wettability on waterflood oil recovery. Journal of Petroleum Science and Engineering 71(34):169-178. https://doi.org/10.1016/j.petrol.2010.01.011.

Zisman, W. A. 1964. Relation of the equilibrium contact angle to liquid and solid constitution. Advanced Chemical Series 43:1-51. doi: 10.1021/ba-19640043.choo1. 


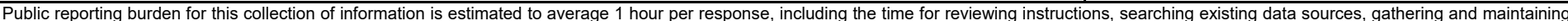

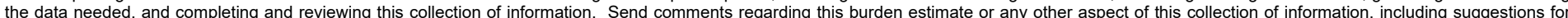

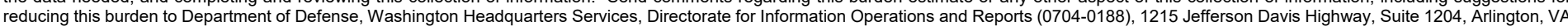

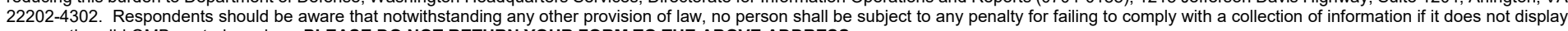
a currently valid OMB control number. PLEASE DO NOT RETURN YOUR FORM TO THE ABOVE ADDRESS.

1. REPORT DATE (DD-MM-YYYY) 1 2. REPORT TYPE June 2019 Final report

\section{TITLE AND SUBTITLE}

Determination of Contact Angle and Surface Tension of Nanomaterial Solutions by Optical Contact Angle System

\section{DATES COVERED (From - To)}

5a. CONTRACT NUMBER

W912HZ-15·2-0032

5b. GRANT NUMBER

5c. PROGRAM ELEMENT NUMBER

\section{AUTHOR(S)}

Qihua Wu, Kathryn Kremer, Stephen Gibbons, and Alan J. Kennedy

5d. PROJECT NUMBER

5e. TASK NUMBER

5f. WORK UNIT NUMBER

7. PERFORMING ORGANIZATION NAME(S) AND ADDRESS(ES)

8. PERFORMING ORGANIZATION REPORT NUMBER

U.S. Army Engineer Research and Development Center, Environmental Laboratory

3909 Halls Ferry Road, Vicksburg, MS 39180-6199

ERDC/EL SR-19-6

\section{SPONSORING / MONITORING AGENCY NAME(S) AND ADDRESS(ES)}

10. SPONSOR/MONITOR'S ACRONYM(S)

Headquarters, U.S. Army Corps of Engineers

Washington, DC 20314-1000

11. SPONSOR/MONITOR'S REPORT NUMBER(S)

\section{DISTRIBUTION / AVAILABILITY STATEMENT}

Approved for public release; distribution unlimited.

\section{SUPPLEMENTARY NOTES}

\section{ABSTRACT}

Contact angle and surface tension measurements reveal wetting characteristics of materials, which are very essential for both fundamental research and manufacturing processes. Among many types of techniques, optical contact angle measurement is considered to be the most convenient method and has been widely used. This scientific operating procedure (SOP) provides general guidance for using an optical contact angle measurement system (e.g., an AST VCA Optima system), for determining contact angle and surface tension of nanomaterials in solution. Procedures and recommendations for sample preparation, instrument preparation, sample analysis, and results analysis are included. This procedure was tested on a variety of nanomaterial dispersions.

\begin{tabular}{|lll}
\hline 15. SUBJECT TERMS & Nanotechnology & Materials \\
& Nanostructured materials--Characterization & Wetting \\
& Surface chemistry &
\end{tabular}

16. SECURITY CLASSIFICATION OF:

\begin{tabular}{|l|l|l|}
\hline a. REPORT & b. ABSTRACT & c. THIS PAGE \\
UNCLASSIFIED & UNCLASSIFIED & UNCLASSIFIED \\
\hline
\end{tabular}

\begin{tabular}{|c|c|}
$\begin{array}{c}\text { 17. LIMITATION } \\
\text { OF ABSTRACT }\end{array}$ & $\begin{array}{c}\text { 18. NUMBER } \\
\text { OF PAGES }\end{array}$ \\
SAR & 28 \\
\end{tabular}

19a. NAME OF RESPONSIBLE PERSON

19b. TELEPHONE NUMBER (include area code) 